\title{
Current Radiation Issues for Programmable Elements and Devices
}

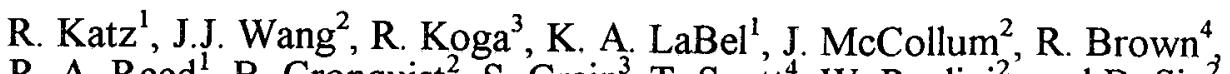

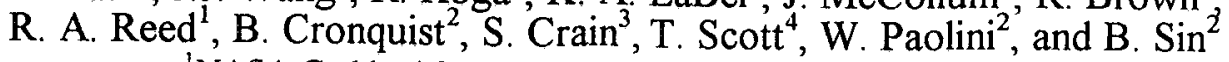 \\ ${ }^{1}$ NASA Goddard Space Flight Center, Greenbelt, MD 20771 \\ ${ }^{2}$ Actel Corporation, Sunnyvale, CA 94086 \\ ${ }^{3}$ The Aerospace Corporation, LA, California 90009 \\ ${ }^{4}$ Lockheed-Martin Federal Systems, Manassas, VA.
}

\begin{abstract}
State of the art programmable devices are utilizing advanced processing technologies, non-standard circuit structures, and unique electrical elements in commercial-offthe-shelf (COTS)-based, high-performance devices. This paper will discuss that the above factors, coupled with the systems application environment, have a strong interplay that affect the radiation hardness of programmable devices and have resultant system impacts in (1) reliability of the unprogrammed, biased antifuse for heavy ions (rupture), (2) logic upset manifesting itself as clock upset, and (3) configuration upset. General radiation characteristics of advanced technologies are examined and manufacturers' modifications to their COTS-based and their impact on future programmable devices will be analyzed.
\end{abstract}

\section{INTRODUCTION}

Programmable logic has become ubiquitous in spacecraft electronic designs as they are inherently flexible and provide systems designers the tools to meet the new trend towards higher integration and performance with decreasing costs and development time. This is not unique to spacecraft electronics as programmable device development is primarily driven by the commercial sector. As such, the devices are becoming increasingly sophisticated architecturally, utilizing unique circuit structures, and are using the most advanced technologies.

Commercial programmable logic devices are utilizing advanced technologies, with manufacturers offering $0.35 \mu \mathrm{m}$ and $0.25 \mu \mathrm{m}$ products, with improved performance levels allowing them to replace ASICs in some applications. For spaceflight electronics, these devices have the potential to efficiently implement circuits in demanding applications such as laser altimetry, photon pulse counting, and time-of-flight electronics.

As the requirements for increased performance drive device development, we see new structures such as phaselocked loops, both digital and analog, as an example. While providing greater system performance by de-skewing clocks and providing clock multiplication capabilities, these structures may be susceptible to new radiation effects. Clock integrity is critical for system applications and we have examined and analyzed several cases of logic upset in clock circuitry. Modifications were required and made to the commercial circuits to reduce device sensitivities to heavy ions.

High-speed interconnections can be made with metal-tometal antifuses, utilizing current processing technologies such as chemical-mechanical polishing (CMP). While these antifuses are biased with relatively low electric field strengths, preliminary studies have shown that these unique structures are susceptible to rupture [1]. Various "recipes" for these elements are explored and data is shown for a highspeed, radiation-hardened antifuse.

FPGAs, in particular, are now using some of the most advanced technologies, scaling internal features and improving performance with some devices operating at lower core voltages. We have seen what is probably the last generation of $5 \mathrm{~V}$ devices being produced at their limit of $0.45 \mu \mathrm{m}$, the $40 \mathrm{MX}$ and $42 \mathrm{MX}$ series. All of the major manufacturers have deep sub-micron devices, generally in the $0.35 \mu \mathrm{m}$ technology, some with $0.25 \mu \mathrm{m}$ features, running at a $3.3 \mathrm{~V}$ core voltage. The state-of-the-art is the announced $0.18 \mu \mathrm{m}$ device from Vantis (AMD), the VFl series; this device runs at a core voltage of $2.5 \mathrm{VDC}$.

The lower core voltages are attractive to system designers because of their high speed and lower power consumption, power being a function of $\mathrm{V}^{2}$. The effects on the SEU and total dose characteristics are studied and are relatively consistent across manufacturers and foundries. Note however, that these advanced devices, to be usable in practical systems, retain an input tolerance of 5.5 volts, a concern for gate rupture in these deep sub-micron processes, requiring special circuit structures to prevent breakdown and conduction to the $\mathrm{V}_{\mathrm{DD}}$ rail.

Configuration upset has been predicted, detected, and analyzed in FPGAs. While the commercial circuit structures are specifically designed for robust behavior in the industrial environment, many design implementations do not handle faults well in the heavy ion environment.

A variety of devices were evaluated in this paper. Some are off-the-shelf production devices; others are either preproduction devices or early prototypes. Applications to a specific product should be done carefully as continuing technological advancements are being made by the manufacturers. 
Pre-Print to be published in the IEEE Transactions on Nuclear Science, December, 1998

\section{RUPTURE}

\section{A. Oxide Nitride Oxide (ONO) Antifuses}

The ONO antifuse is frequently used in non-volatile space flight applications. Biased, unprogrammed antifuses are susceptible to heavy ion-induced rupture and improvements have been made in antifuse design [1]. Recent testing has augmented our data set for the hardened antifuse in the RH1020, with the production devices' antifuses having a $90 \AA$ thickness. Results are shown in Figure 1. The beam was normal to the die, worst-case, and a fluence of $10^{7}$ ions $/ \mathrm{cm}^{2}$ used for each run. A significant positive margin exists above a bias voltage of $5.5 \mathrm{VDC}$ at an LET of $37 \mathrm{MeV}-\mathrm{cm}^{2} / \mathrm{mg}$. This is adequate for most missions and the antifuse can be classified as radiation-tolerant; it has improved radiation performance over its commercial ancestors.

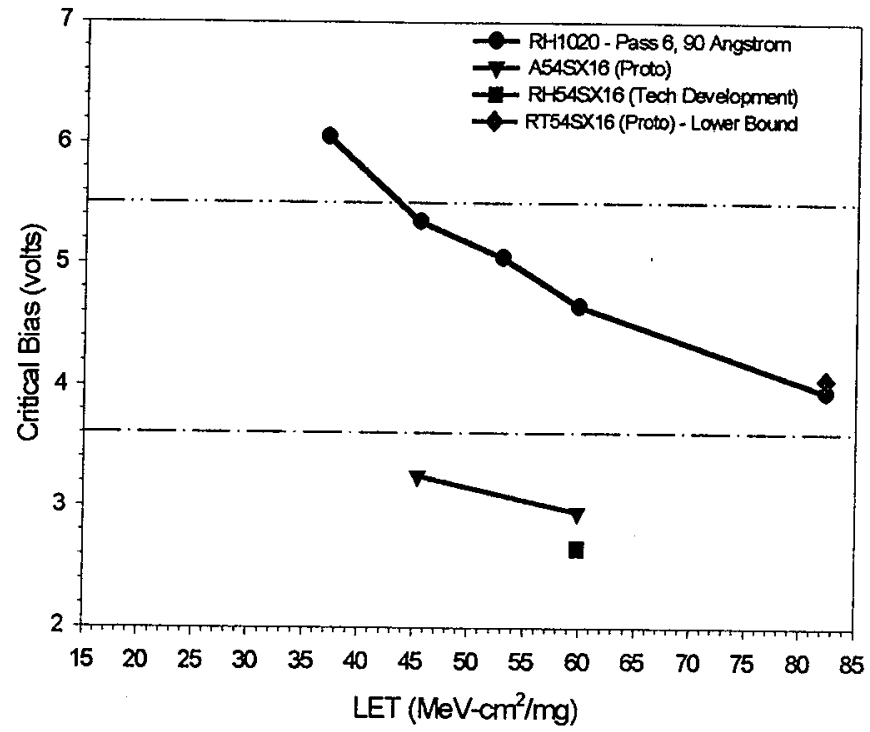

Figure 1. Summary of antifuse rupture data. Positive margin at $\mathrm{LET}=37 \mathrm{MeV}-\mathrm{cm} 2 / \mathrm{mg}$ is shown for production $\mathrm{RH} 1020$ with a hardened ONO antifuse. One "recipe" of an M2M antifuse did not fail at LET $=82 \mathrm{MeV}-\mathrm{cm} 2 / \mathrm{mg}$, $\mathrm{V}_{\mathrm{BIAS}}=400 \mathrm{mV}$.

\section{B. Metal-to-Metal (M2M) Antifuses}

Metal-to-metal antifuses hold performance advantages over the ONO version; programmed resistance's are $\sim 25-50$ ohms versus $\sim 100-500$ ohms for FPGA implementations. M2M antifuse resistance is $<1 \mathrm{ohm}$ for the less densitycritical programmable substrates. These devices are enabled by advances in processing technologies that are critical for controlling the thickness of the programmable elements. References [1-3] discuss their hardness to total dose effects.

While ONO antifuse thickness is of the order of $100 \AA$, M2M antifuse thickness typically varies from $500 \AA$ to $1000 \AA$, resulting in a decreased electric field strength of a factor of 5 to 10 , assuming equal bias voltages. This assumption is not valid for current devices. Programmable substrates program at approximately $30 \mathrm{VDC}$ supporting analog supply voltages of $\pm 12 \mathrm{VDC}$; careful routing ensures that no antifuse sees more than a $12 \mathrm{VDC}$ bias. Recent M2M antifuse FPGAs operate at lower voltages, further decreasing the electric field strength in the biased antifuse by $35 \%$. Reliability studies conducted on M2M antifuses $[2,4,5]$ show MTBF as a function of bias voltage.

Antifuse rupture data is a function of heavy ion LET and electric field strength [1] and the failure points of some of the amorphous silicon antifuses occur at a far lower electric field strength than either the ONO antifuses or the $\mathrm{SiO}_{2}$ reference data [6]. Failure analysis, using several different techniques, has been conducted, assisting in the determination of failure and investigation of the mechanisms. Emission microscope techniques have proven successful for ONO antifuse and a liquid crystal methodology for a variety of M2M damaged antifuses. In addition, current-voltage curves for damaged antifuses vary by construction.

To expand on our results, a next generation DUT card has been built with new devices designed and fabricated for M2M antifuses. Devices that have been tested include the QL3025, prototype A54SX16, RT54SX16, and the RH54SX16's, and the UT22VP10. The QL3025 is a $3.3 \mathrm{~V}$, $0.35 \mu \mathrm{m}$ device fabricated at TSMC. Each of the SX prototypes uses a different antifuse "recipe" for these experiments. The A54SX16 is also a $3.3 \mathrm{~V}, 0.35 \mu \mathrm{m}$ device while the RT54SX16 and the RH54SX16 are both $3.3 \mathrm{~V}$, $0.6 \mu \mathrm{m}$ devices. These devices all have 5 volt-tolerant $\mathrm{I} / \mathrm{O}$, supported by a $5 \mathrm{~V}$ bias, and have a $3.3 \mathrm{~V}$ core. The UT22VP10 is a $5 \mathrm{~V}$ PAL; the test data for this device was inconclusive and will not be discussed in this paper. The QL3025 had significant latchup problems, preventing an analysis of their antifuse structure.

Results for the three SX M2M antifuse prototypes is shown in Figure 1 where the bottom reference line is set at 3.6 volts, the maximum rated voltage for this technology. At a LET of $37 \mathrm{MeV}-\mathrm{cm}^{2} / \mathrm{mg}$, all of the devices showed significant positive margins, with no damage detected, giving SEU-tolerant performance. At the higher LETs, differences are observed between the three variants, with two of the devices failing at an LET of $45 \mathrm{MeV}-\mathrm{cm}^{2} / \mathrm{mg}$.

The significant result is the performance of "recipe M." Several lots were tested, showing radiation-hard performance, no damage at an LET of $82 \mathrm{MeV}-\mathrm{cm}^{2} / \mathrm{mg}$. Additional margin for this design was demonstrated by increasing the bias voltage to $4.0 \mathrm{VDC}, 11 \%$ greater than the rated maximum. Further experiments are planned which will test the effects of different variants of the recipes to give further insight into the mechanism.

Testing techniques are similar for the ONO and M2M antifuses although damage to the M2M-based structures are far easier to detect. These structures, designed for lower programmed impedances, also have lower impedances when damaged by a heavy ion. Despite the higher bias across the ONO structure, the current draw by a damaged M2M structure is many times higher. 
Pre-Print to be published in the IEEE Transactions on Nuclear Science, December, 1998

\section{LOGIC UPSET}

\section{A. Introduction}

Logic upset is s a transient pulse from a single ion strike. This can occur in combinational circuits or global resources such as clock and reset lines. Previously, logic upset was observed in an FPGA but cross-sections were not determined.

\section{B. Logic Upset Examples and Instrumentation}

Gate upsets in the RH1280 were observed when running at a reduced supply voltage of $3.3 \mathrm{VDC} \pm 10 \%$; there were no logic upsets when running at supply voltages $\geq 4.5 \mathrm{VDC}$. Failures occurred in the voter circuits of TMR strings and correlation with on-chip error monitors confirmed logic upset. This is an example of a data upset, with the glitch or runt pulse arriving at the input to the flip-flop during the critical time when the data is latched, typically a small fraction of a nanosecond. There was no effort to harden the logic circuits of the RH1280, originally a commercial design.

The CX2041 $(0.6 \mu \mathrm{m})$, was evaluated for SEE at a bias of 3.3 VDC. In this device, a low-skew clock tree is built and balanced to support user flip-flops. During heavy ion irradiation, large numbers of errors appeared in bursts with some errors disrupting the entire chip, indicating disruption to the clock distribution network. Errors were observed simultaneously in several independent shift register strings, with the only common element being the clock tree. Additionally, few errors were reported on the SEU monitor of our SEU-hardened shift register [1], while the shift register itself gave burst errors. This is an example of a clock upset.

The DUT stimulus pattern is critical for detecting clock upsets: all ' 1 's or all ' 0 's are useful for detecting pattern sensitivity in flip-flops but will mask clock upsets since an extra clock will be undetected. Similarly, a Johnson twisted ring counter, with at most one ' 1 ' - ' 0 ' transitions, will have trouble distinguishing clock upsets from SEUs.

SEU time-tagging has been added to our test equipment, permitting both SEUs and logic or clock upsets to be measured simultaneously. Sample output from an RH1020 run is shown in Figure 2, where error counts for each of the three monitors is plotted against sample number, with approximately $250 \mu$ s between sampling points. The unhardened shift register's error counts (DOS) rise linearly and then jump approximately 17 counts, one-half the length of the shift register. Concurrent with this jump, the hardened output's error count (DOH) undergoes a similar jump and stays flat, as it rejects SEU's. Note that the hardened shift registers SEU monitor (TMR MON) never jumps, and rises linearly with time, showing a relatively constant error rate in the flip-flop elements. By taking the first derivative of the error count with respect to sample number, the number of clock upsets may be counted and the number of upsets attributed to clock upset may be removed from the total error count, permitting an accurate assessment of flip-flop SEU sensitivity as shown in Figure 3.

\section{RH1020 Logic Upset Analysis and Mitigation}

The clock upset cross-section in pre-production models of the RH1020 is significantly higher than that of it's commercial cousin, the A1020B (1.0 $\mu \mathrm{m}$ device). This is thought to be attributed to moving the design to the higher performance radiation-hardened process, where small runt pulses propagate easier and flip-flops respond to signals with a smaller pulse width and amplitude [7].

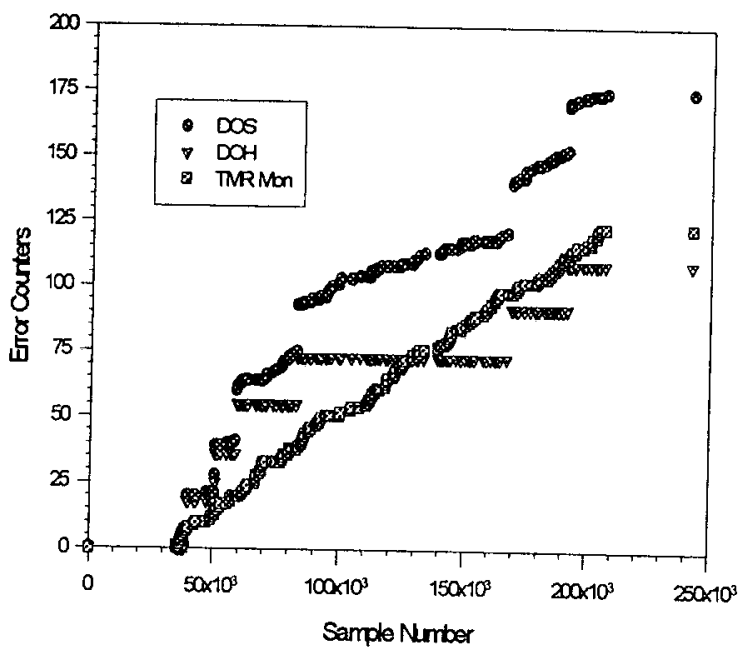

Figure 2. Clock upset in the RH1020 with each SEU timetagged. The concurrent jumps on DOS and DOH, two independent circuits, indicate clocks upset, as the clock is the common element

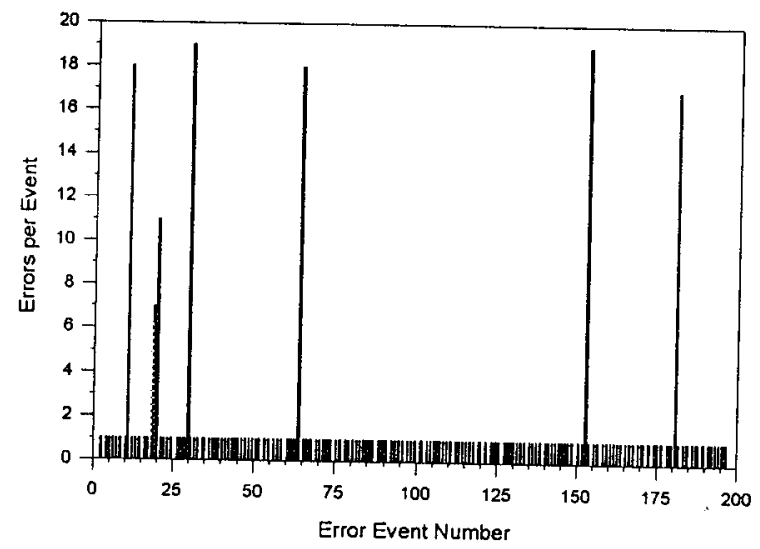

Figure 3. Quantitative analysis of clock upset raw data It is performed by plotting the sample-to-sample differences in the errors counters

Although a channeled architecture with individual buffers and clock lines for each row, the A1020B/RH1020 shorts the outputs of the buffers together to minimize clock skew, resulting in many common points to the distribution network. The earlier Al020 $(2.0 \mu \mathrm{m})$ and Al020A $(1.2 \mu \mathrm{m})$ devices had isolated row buffers and similar error signatures indicated that the upsets were present in the input stage, not in one of the clock row drivers. A number of analytical and experimental techniques such as laser stimulation and using a focused ion beam (FIB) to perform circuit modifications 
Pre-Print to be published in the IEEE Transactions on Nuclear Science, December, 1998

further isolated the sensitive region to a TTL-compatible input stage employing a small amount of hysteresis.

Analysis of transistor sizes and clock upset cross-sections led to the preliminary conclusion that the upset was happening on the clock's transition. Several experiments were run to verify this. During heavy ion irradiation, the input was held at either rail, with few clock upsets detected with the clock input grounded. This dependence is consistent with transistor-level circuit analysis, which showed a strong asymmetry, typical of TTL-level inputs, and laboratory noise testing on the input stage. This conclusion was confirmed with heavy ion irradiation by measuring the frequency dependence of the upset as shown in Figure 4, with four devices tested from $10 \mathrm{kHz}$ to $1 \mathrm{MHz}$, each run having a fluence of $10^{7}$ ions $/ \mathrm{cm}^{2}$. The large part-to-part variation in Figure 4 is typical for this effect, as the propagation of a clock runt pulse and acceptance by a flip-flop is marginal and dependent on specific device parameters. Analysis of the number of upsets per hit, usually about one-half of the shift register length, confirms this as a runt pulse, consistent with other laboratory tests and circuit analyses.

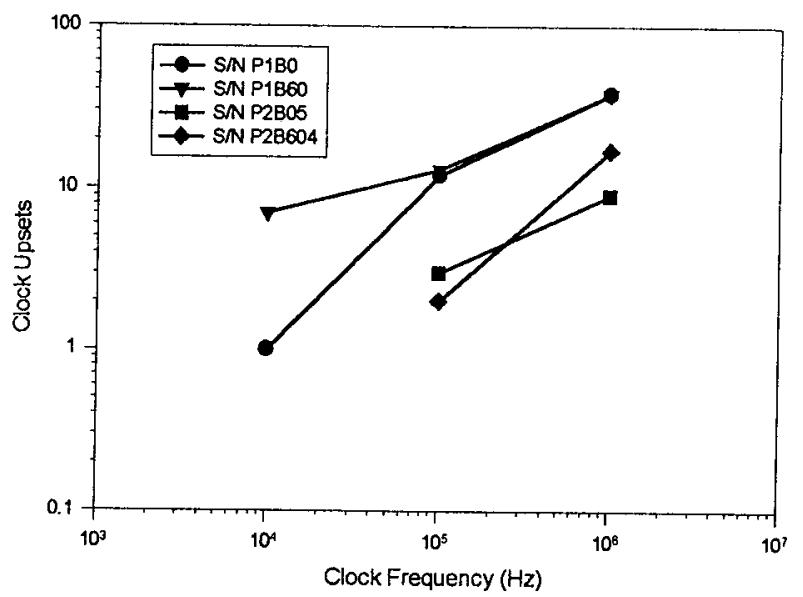

Figure 4. RH1020 (pre-production devices) input buffer transients resulting in "clock upsets." The upset occurs on the transition and is shown here as a frequency dependence

While this phenomenon has been referred to as "clock upset," we refer to it in the more general sense as "logic upset." An examination of the schematics for non-dedicated LOO stages showed a similar circuit construction to the dedicated clock input pin on the 1020 series. A special DUT was designed and constructed to verify this $\mathrm{I} / \mathrm{O}$ module's sensitivity. Since regular I/O pins in the RH1020 do not permit access to a low-skew signal distribution network, flipflops were hand-placed so that the clock input for each of the flip-flops was attached to the same routing segment and no skew was confirmed with the static timing analyzer. While detailed cross-sections were not measured as a result of limited test time, upset on these $\mathrm{V} / \mathrm{O}$ pins was detected, confirming the analysis' conclusion that the fault was in the input stage and not in the clock distribution network. These "non-clock" pins will show an edge SEU sensitivity since typical circuit applications such as ripple event counters use these pins as clocks.

Based on circuit analysis and SPICE simulations, along with the heavy ion. laser, and laboratory tests on A1020, A 1020A, A1020B, and RH1020 (including those with modified circuits using the FIB), a new production lot was fabricated, with the original commercial TTL-compatible input circuit design modified to mitigate the logic upset. While the cross-section of the original devices was low, approximately $10^{-6} \mathrm{~cm}^{2} /$ Clock, the modifications resulted in a reduction by an order of magnitude. Figure 5 shows the results, plotting both typical pre-production data vs. an average of five production devices. Clock upset was also detected in the A1020, A1020A, A1020B, and CX2041 microcircuits.

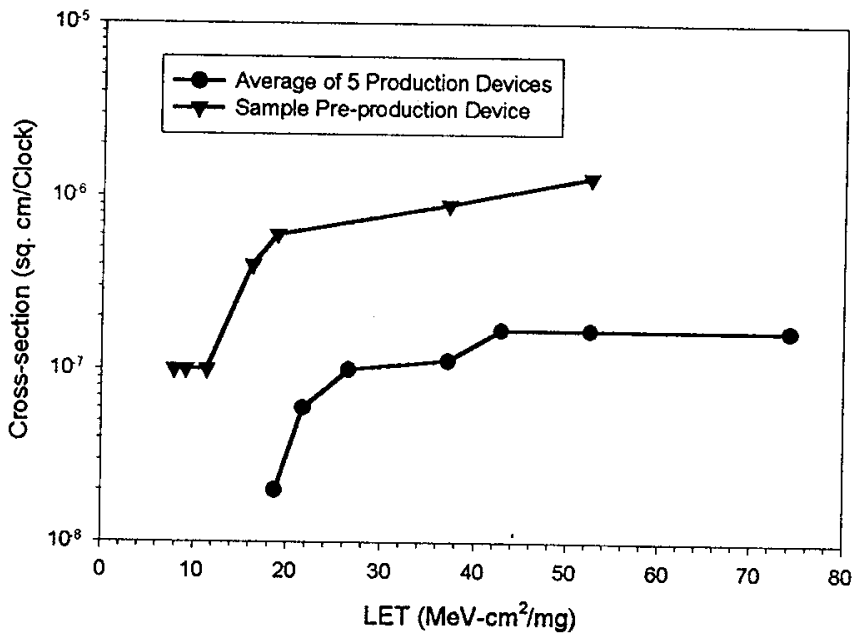

Figure 5. Clock upset performance of pre-production and production RH1020 devices. The production devices had a modified input circuit for improved SEE performance

\section{CONFIGURATION UPSET}

\section{A. Introduction}

For many types of microcircuits, the mode and the control of the chip may be configured in a variety of ways. The configuration of a field programmable gate array may be controlled by an antifuse, an SRAM cell, a non-volatile memory cell, or a combination of these technologies. Advanced microcircuits may contain various test modes as well as functions reserved for the manufacturer, such as device identification or programming.

Configuration bits may be susceptible to SEUs and a loss of control of the integrated circuit may result. Reference [8] gives a good overview of this type of effect and shows failure modes for an AT28C010 EEPROM, which fall into two classes of failures. One class corresponds to an SEU in the output data register. A second class, consisting of two distinct sub-classes, led to a semi-permanent loss of control and an increase of supply current. 
Pre-Print to be published in the IEEE Transactions on Nuclear Science, December, 1998

\section{B. Analysis of a Configuration Upset}

Tests were run on prototype FPGAs. The devices would, when irradiated with heavy ions, apparently lose all functionality until the power was cycled, accompanied by changes in the device current. The following section will determine the failure mechanism as well as discuss test and analysis techniques. These prototype devices implement a new architecture for this manufacturer with test samples utilizing two different scaling factories and three different foundries.

A representative run showing one class of error signatures is shown in Figure 6. A 5 V bias is used for 5 volttolerant $\mathrm{I} / \mathrm{O}$ and a $3.3 \mathrm{~V}$ bias powers the array core and output drivers. Large currents were observed and remained until the device's power was cycled. In some cases, the current draw exceeded $800 \mathrm{~mA}$, the programmed setting for the power supply. Although this current rise is often a result of SEL, no determination was immediately made. Similar results were seen on devices from several foundries and the device irradiated in Figure 6 was from a radiation-hardened line using a $2 \mu \mathrm{m}$ epitaxial layer. The design rules for the device were consistent with good latchup performance and the small failure cross-section suggested a different mechanism. Other failure signatures were observed, again with an apparent loss of functionality. One class shows the current levels dropping from their active state to quiescent levels.

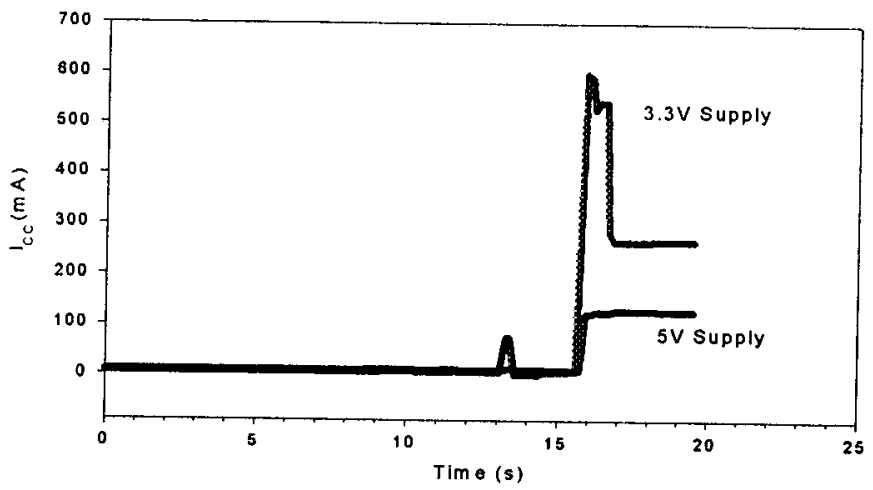

Figure 6. Strip chart during irradiation of a prototype FPGA produced on a radiation-hardened, $2 \mu \mathrm{m}$ epi line. The current on some runs exceeded $800 \mathrm{~mA}$ and was caused by a configuration error from an SEU in the 1149.1 TAP controller.

It has been determined that these two cases have a common cause, and is a result of the IEEE JTAG 1149.1 TAP [9] specification and implementation. This standard specifies the interface and operation of features to support test and other operations at the board and device level.

An overview of the '1149.1' scan configuration is shown in Figure 7, with a set of cells between the device's pins and the internal core logic. This permits various operations such as sampling the devices inputs, driving the external pins to known values, or presenting test inputs to the device core. The scan cells form a data register and are configured as a shift register. Other 1149.1 functions include device identification and other device specific features such as builtin self test (BIST), programming, etc.

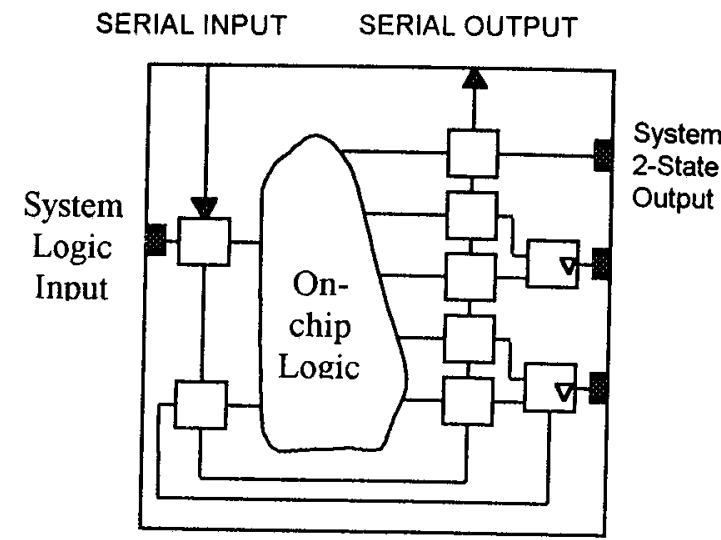

Figure 7. The IEEE JTAG 1149.1 scan architecture. The logic core is surrounded by the scan cells, which can perform normal IO functions or be controlled from the test access port (TAP).

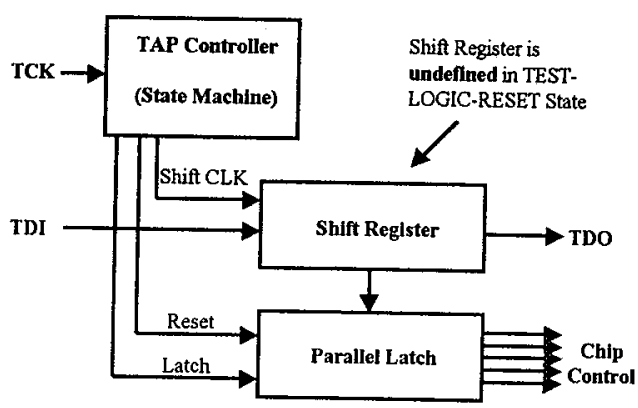

Figure 8. An SEU in the TAP controller. Undefined data is loaded into the output of the Instruction Register causing an apparent loss of functionality.

The test access port (TAP) controller commands the test interface and registers with a sixteen-state sequencer. Figure 8 shows a simplified diagram of the basic configuration as it is typically implemented, without the hard reset pin (TRST!, which is optional in the standard), along with the instruction register. The output of the instruction register controls the device and consists of two sections, a shift register for serial loading and a parallel latch to hold the instruction, which is loaded and cleared on command from the TAP controller. The JTAG nomenclature defines TCK as the clock to the TAP controller, TDI is test data in, and TDO is test data out. TMS (mode pin. not shown) guides transitions in the TAP controller.

State assignments for the TAP controller may be arbitrarily selected with the states and transitions rigidly defined in the specification. The Test-Logic-Reset state is used for normal operations with the TAP controller asserting RESET. forcing the output latch to load an instruction which does not affect the device's operation. An analysis of the TAP controller's state diagram shows a well-designed, robust controller for commercial and industrial applications, tolerant of faults that would be expected in these environments (such as pins being shorted) and no lock-up states. All paths will return the machine to the Test-Logic-Reset state within 5 TCK cycles with TMS held high. However, when analyzing 
the state diagram for SEU sensitivity, the machine will return to the Test-Logic-Reset state, but, if the appropriate state bit is upset, via a path that moves through Update-IR, which latches new data into the instruction register's latch, giving the microcircuit a new command mode or configuration. Analysis of the state assignment used in the prototype devices tested showed that a single bit error could force sequencing through the Update-IR state.

Note in Figure 8 that the shift register located in the instruction register is not held in reset when the TAP controller is in the Test-Logic-Reset state. This is consistent with the 1149.1 specification, which states that the shift register is undefined in that state, amongst others, with the authors of the specifications not anticipating SEUs. When an SEU flips a bit in the TAP controller and the Update-IR is traversed, arbitrary values are loaded into the IR register, with an unpredictable effect on the chips operation and state.

The small number of susceptible bits in the TAP controller is consistent with the failure rate during heavy ion irradiation. However, latchup was not immediately ruled out, as we kept open the possibility that a design rule may have been violated in one spot which was not caught by the design rule checker. The next experiment used a $6 \mathrm{kHz}$ clock driving the TCK pin with TMS held high. $6 \mathrm{kHz}$ was selected to facilitate instrumentation while having a minimal probability of two upsets in the 5 TCK sequence. In this mode the device experienced functional failures during irradiation but power cycling was not required to restore the device to an operational mode, as would be required after a SEL. Note that each device must be analyzed to ensure that damage can not result from operation in this mode and that a higher frequency for TCK should be used if devices with this design are flown.

The failure mechanism was further investigated by placing the instrumentation in time-tagging mode with the $6 \mathrm{kHz}$ signal driving TCK. Sample results are shown in Figure 9, with the outputs of error counters shown for the two independent flip-flop strings, sampled at approximately $4 \mathrm{kHz}$. Three jumps are seen, with both error counters changing simultaneously and in equal amounts, similar to that seen from a clock upset, discussed above in Section III.

A closer examination and further tests with various DUT stimulus proved that this similar signature could not have been clock upset. Examination of the error counts showed sizes that were too large to suggest clock upset for the length of the shift registers and clock frequencies used. However, a brief oscillation of the clock buffer could not be ruled out, with perhaps multiple transitions. The next test was to decrease the TCK frequency by an order of magnitude and observe the frequency of configuration losses and the magnitude of the jumps. By examining the error counts, which are based by the $1 \mathrm{MHz}$. DUT clock, the period of time of the malfunction can be determined precisely. This, coupled with decreasing the TCK frequency by an order of magnitude, resulted in the rate of configuration upsets remaining constant but the size of the jumps increased. The longer periods of loss of functionality would not have occurred in clock upset. which is an asynchronous affect. Lastly, additional runs and changes in the input patterns resulted in data sets where only one of the two shift registers experienced the jumps in error counts. In this mode, by holding the data constant, for example, a matching value in the boundary scan register would show no errors while a fault was occurring.

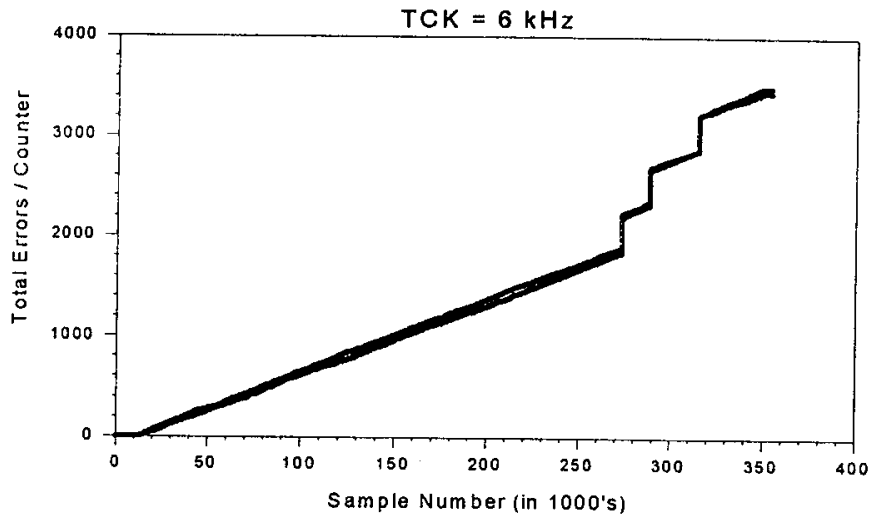

Figure 9. Transient functional failures of a prototype FPGA incorporating 1149.1 circuitry. The duration of the failure is a function of the TCK frequency.

\section{RADIATION TEST RESULTS AND ANALYSIS}

This section will summarize, discuss, and analyze recent radiation test results of a more general character than the sections above. Tests continue on existing programmable products to support on-going flight programs and emerging trends. On the advanced technology front, the first deep submicron programmable data is presented which show radiation-tolerant performance for some parameters. With slight modifications made to the commercial foundry's process, radiation-hard performance levels were obtained.

\section{A. Heavy Ion and Proton Induced SEU}

\section{1) Heavy Ion Effects and Analysis}

The RH1020, which is based on the A1020B commercial design, has recently been characterized for SEU performance. The SEU performance of the A1020B is heavily influenced by the Act 1 architecture, which contains no hard-wired flipflops; latches are made by feeding back the output of the $4: 1$ multiplexor element back to its input via the routing channels and through antifuses. Radiation-tolerant performance is obtained since the pre-laid routing tracks have a parasitic capacitance and the antifuses have parasitic resistance which act as a filter. The weakness of this architecture with respect to SEU is the design of the module resulting in very asymmetrical behavior, an artifact of its commercial origins, with the weaker state dominating the upset rate. The RH1020, fabricated on the Lockheed-Martin Federal Systems (LMFS) $5 \mu \mathrm{m}$ epitaxial process would be expected to have similar SEU performance to its commercial equivalent. The data shown in Figure 10 shows this. Similarly, the Act 2 
Pre-Print to be published in the IEEE Transactions on Nuclear Science. December, 1998

A1280A and RH1280 show similar heavy ion SEU performance.

A similar analysis can be applied to two quick-turn ASIC families, the QYH500 $(0.8 \mu \mathrm{m})$ and the CX2001 $(0.6 \mu \mathrm{m})$, produced by Chip Express. Neither series contains hardwired or dedicated flip-flops with the NAND-based QYH500 giving superior SEU performance to the coarser grained, multiplexor-based CX2001. Sensitive circuit nodes internal to the CX2001 logic module may be hit by an SEU, without the parasitic capacitance of a routing track adding to the storage elements SEU response time as in the QYH500, which has excellent SEU characteristics. It is interesting to compare the CX2001 and Act 1 architectures, which are very similar, and it can be expected that the mechanism leading to the vulnerability of the Act 1 module leads to the SEU performance of the CX2001 module.

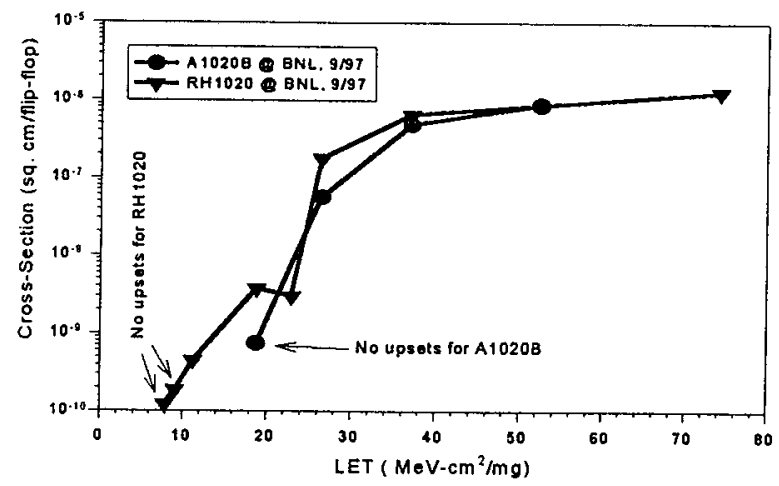

Figure 10. Comparison of SEU performance for devices fabricated at commercial (MEC) and radiation-hardened (LMFS) foundries.

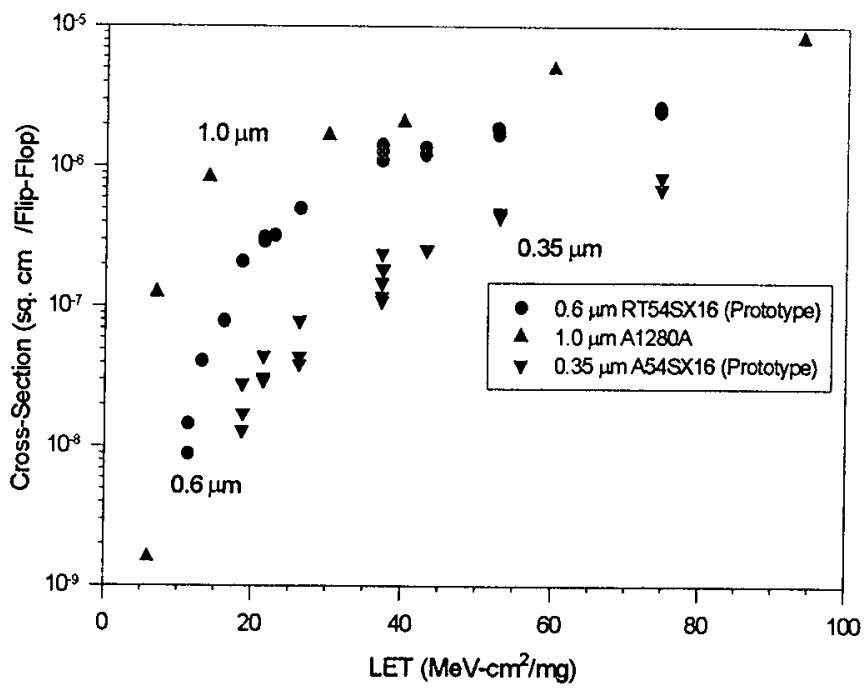

Figure 11. SEU Response of hard-wired flip-flops at $1.0 \mu \mathrm{m}$, $0.6 \mu \mathrm{m}$, and $0.35 \mu \mathrm{m}$ feature sizes. The $1.0 \mu \mathrm{m}$ device has a nominal $5 \mathrm{~V}$ bias; the others have a $3.3 \mathrm{~V}$ nominal bias

Utilizing prototype RT54SX16 and A54SX16 devices, we can further measure and analyze the SEU performance of $3.3 \mathrm{~V}$ technology components down to $0.35 \mu \mathrm{m}$ feature sizes. Last year's study [1] of $0.6 \mu \mathrm{m}$ devices utilized a small pattern oriented towards reliability testing; this study uses pre-production devices with 400 flip-flops per DUT, giving more accurate thresholds and cross-sections with the results summarized in Figure 11. Both of the $3.3 \mathrm{~V}$ sea-of-modules devices, with the more symmetrical circuit designs,

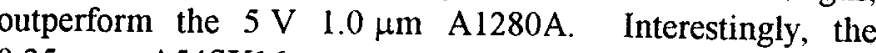
$0.35 \mu \mathrm{m} \quad$ A54SX16 prototype outperforms the $0.6 \mu \mathrm{m}$ RT54SX16 prototype with both devices having identical test patterns programmed.

\section{2) Proton Effects and Analysis}

A number of programmable devices have been tested for proton sensitivity and Table 1 gives estimates of crosssections for approximately $195 \mathrm{MeV}$ protons. The A1280A has been tested previously. A review of the data and test plan, along with the low heavy ion upset threshold for the hard-wired flip-flops, led us to repeat this test using an array of 19 devices from four different production lot date codes. Each of these devices upset, with runs being conducted at both the worst-case 4.5 VDC and nominal 5.0 VDC supplies. The A1280A results are discussed in detail in [10].

Table 1. Proton Sensitivities at $195 \mathrm{MeV}$

\begin{tabular}{|l|l|l|l|}
\hline Device Type & $\begin{array}{c}\text { Size/Voltage } \\
\text { (nominal core) }\end{array}$ & \multicolumn{1}{|c|}{$\begin{array}{c}\text { Est. X-Sec } \\
\left(\mathrm{cm}^{2} / \mathrm{f}-\mathrm{f}\right)\end{array}$} & Comments \\
\hline A1280A & $1.0 \mu \mathrm{m} / 5.0 \mathrm{~V}$ & $\sim 137 \times 10^{-15}$ & 19 devices \\
\hline RH1020 & $1.0 \mu \mathrm{m} / 5.0 \mathrm{~V}$ & $<2 \times 10^{-15}$ & \\
\hline RH1280 & $0.8 \mu \mathrm{m} / 5.0 \mathrm{~V}$ & $\sim 400 \times 10^{-15}$ & S-Module \\
\hline QYH500 & $0.8 \mu \mathrm{m} / 3.3 \mathrm{~V}$ & $<0.5 \times 10^{-15}$ & No upsets \\
\hline RT54SX16 & $0.6 \mu \mathrm{m} / 3.3 \mathrm{~V}$ & $\sim 6 \times 10^{-15}$ & \\
\hline QL3025 & $0.35 \mu \mathrm{m} / 3.3 \mathrm{~V}$ & $<4 \times 10^{-15}$ & No upsets \\
\hline A54SX16 & $0.35 \mu \mathrm{m} / 3.3 \mathrm{~V}$ & $\sim 3 \times 10^{-15}$ & \\
\hline JT22VP10 & $\mathrm{N} / \mathrm{A} / 5.0 \mathrm{~V}$ & $\sim 2 \times 10^{-11}$ & Cypress die \\
\hline
\end{tabular}

The behavior of the radiation-hardened FPGAs is examined for proton upset. The RH1280, based on the commercial A1280XL, has a relatively large upset crosssection, as the device was not modified for SEU hardness. The RH1020, based on the commercial A1020B, is also susceptible. The $3.3 \mathrm{VDC}$ small feature-sized commercial devices did relatively well, with upset crosssections having the same order of magnitude as the $5.0 \mathrm{~V}$ RH1020 which utilizes routed flip-flops. No upsets were detected in the QL3025's, consistent with the heavy ion tests, where no upsets were detected with ions having a LET of $18.8 \mathrm{MeV}-\mathrm{cm}^{2} / \mathrm{mg}$, although total fluence was low because of SEL problems. The cross-sections of the prototype RT54SX16 and the A54SX16 are low, consistent with the heavy ion data of Figure 11.

Lastly, as seen in Table 1, no upsets were detected for QYH530 ASICs, with devices selected from two one-mask production lots. The devices in this test configuration were operated at 3.3 volts.

\section{3) SR-1M Configuration Memory Analysis}

SRAM-based FPGAs are configured by loading state information into SRAM cells are popular commercially and, 
Pre-Print to be published in the IEEE Transactions on Nuclear Science, December, 1998

as discussed below, are the subject of several development efforts for space flight electronics. These SRAM-based devices currently hold a density edge for commercial devices. even taking various gate counting methodologies into account. However. for a radiation-hard device utilizing existing architectures, there will, for the near-term at least, be restrictions on device density.

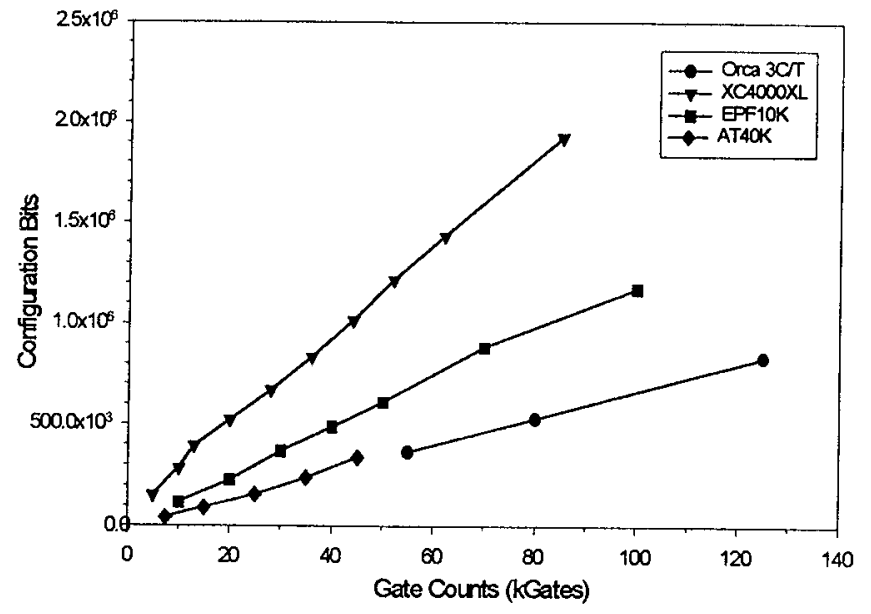

Figure 12. Number of configuration bits vs. gate count for four families of FPGAs. Architectures show different "efficiencies" per configuration bit.

The commercial SRAM-based FPGA vendors each take a differing approach to their architecture, offering structures such as look up table (LUT)-based and logic-based modules, different module granularity, etc. An analysis of almost all available and announced devices shows that the ratio of user flip-flops to user on-chip SRAM to configuration memory is a constant, with approximately an order of magnitude between each storage type. Focusing on the configuration memory bits that dominate, we can see the linear increase in configuration storage as a function of device capacity in Figure 12. The slopes of these curves gives a measure of the configuration bit efficiency for each architecture that is critical for assessing the impact of producing a radiation-hardened device. Even at the $100 \mathrm{kgate}$ density level, we can see a range of configuration bits from below $500 \times 10^{3}$ to almost $2 \times 10^{6}$. Extrapolating to higher device densities, it is clear that radiation-hardened devices are not practical for state of the art commercial technologies. SEU-tolerant applications, as discussed in [1], would employ checking circuits to verify the contents of configuration memory and take corrective action in the case of a fault. It would have to be shown that no permanent circuit damage would occur. The system design would have to be tolerant of a pause in circuit operation if a reload or partial reload is necessary and prevent any erroneous signals from propagating to a critical part of the system. As device geometries continue to shrink, reducing the 'cost' of the silicon, it may become feasible to provide a TMR plus voter for each configuration bit with a nonintrusive, on-chip background process scrubbing the configuration memories.

\section{4) Mitigation Technology}

TMR for user FPGA circuits has been discussed and analyzed in [1] and while not resource efficient, it is used for the effective SEU-hardening of flip-flops. As the commercial industry has moved toward hardware description languages (HDLs) such as VHDL. designers were locked into tedious schematic-based solutions. Over the past year, modifications and scripts for commercial software have been released, offering a choice of HDL tools as well as macro generators and custom schematic libraries. Typically, the designer may select levels of SEU hardening, such as limiting flip-flop selection to "C-Modules" or by selecting TMR structures.

\section{B. Single Event Latchup}

Recent latchup test results are shown in Table 2. Destructive effects were observed for the two Quicklogic devices, the QL24x32 and the QL3025. The $0.65 \mu \mathrm{m}$ series Cypress device had been tested previously with similar results. The QL3025 is a member of the new pASIC3 family, utilizing a $0.35 \mu \mathrm{m}$ TSMC process.

Quick-turn ASIC prototypes are configured by 'laser programming' while flight devices are processed with a onemask technology. The one-mask QYH530's performed similar to the LPGA devices which were tested in [1], with SEL LET thresholds of approximately $60 \mathrm{MeV}-\mathrm{cm}^{2} / \mathrm{mg}$. The thin-epi CX2041 LPGA devices easily latched, both at $\mathrm{VCC}=5.0$ and 3.3 volts. Recent testing did not detect latchup at a LET of $37 \mathrm{MeV}-\mathrm{cm}^{2} / \mathrm{mg}$ when biased at 2.5 volts.

Lastly, while commercial XC3090's had a SEL threshold of approximately $4-7 \mathrm{MeV}-\mathrm{cm}^{2} / \mathrm{mg}$ [1], the specially processed XQR4036XL prototype showed no latchup at an LET of $100 \mathrm{MeV}-\mathrm{cm}^{2} / \mathrm{mg}$ at a temperature of $125^{\circ} \mathrm{C}$, showing radiation-hard performance for this parameter [11].

Table 2. Latchup Summary

\begin{tabular}{|c|c|c|c|}
\hline Device Type & $\begin{array}{l}\text { Size/Voltage } \\
\text { (nominal core) }\end{array}$ & $\begin{array}{c}\text { Threshold } \\
\left(\mathrm{MeV}-\mathrm{cm}^{2} / \mathrm{mg}\right)\end{array}$ & Comments \\
\hline RH1020 & $1.0 \mu \mathrm{m} / 5.0 \mathrm{~V}$ & $>74$ & \\
\hline QL24×32B & $0.65 \mu \mathrm{m} / 5.0 \mathrm{~V}$ & $<18$ & Destructive \\
\hline RT54SX16 & $0.6 \mu \mathrm{m} / 3.3 \mathrm{~V}$ & $>82$ & \\
\hline QYH530 & $0.8 \mu \mathrm{m} / 5.0 \mathrm{~V}$ & $\sim 52$ & One-Mask \\
\hline CX2041 & $0.6 \mu \mathrm{m} / 2.5 \mathrm{~V}$ & $>37$ & LPGA \\
\hline A54SX16 & $0.35 \mu \mathrm{m} / 3.3 \mathrm{~V}$ & $>74$ & \\
\hline QL3025 & $0.35 \mu \mathrm{m} / 3.3 \mathrm{~V}$ & $<11$ & Destructive \\
\hline $\mathrm{XQR} 4036 \mathrm{XL}$ & $0.35 \mu \mathrm{m} / 3.3 \mathrm{~V}$ & $>100$ & \\
\hline
\end{tabular}

\section{Total Dose}

Lot-specific total dose testing is used to support on-going NASA flight programs. The $1.0 \mu \mathrm{m} \mathrm{A1280A/MEC} \mathrm{and} \mathrm{the}$

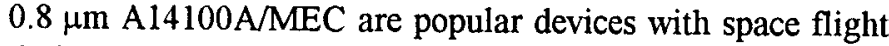
designers utilizing commercial/military-grade technologies. We have seen performance for the A1280A and the A14100A (Act 3) of about 7-10 krads (Si) and greater than 20 krads (Si) respectively. Recent test data show the start of a trend, with severely decreased TID performance for recently fabricated Al280A. AI460A, and A14100A die. 
Pre-Print to be published in the IEEE Transactions on Nuclear Science. December, 1998

Recent data is shown in Figure 13 for new $0.35 \mu \mathrm{m}$ $3.3 \mathrm{VDC}, 0.45 \mu \mathrm{m} 5.0 \mathrm{VDC}$, and $0.6 \mu \mathrm{m} \mathrm{3.3} \mathrm{VDC}$ devices. The $5 \mathrm{~V} \mathrm{~A} 42 \mathrm{MX} 09$ did poorly at about 6 krads (Si) while the $3.3 \mathrm{~V}$ core devices all showed solid radiation-tolerant performance. Of note is the specially processed $0.35 \mu \mathrm{m}$ $\mathrm{XQR} 4036 \mathrm{XL}$, which passed the $60 \mathrm{krad}(\mathrm{Si})$ point with no parametric or functional failures. Devices have been submitted for product analysis as it is thought the reduction in specific structure dimensions has played a role in the increased total dose performance of these four $3.3 \mathrm{~V}$ device types, clearly indicating a trend. $0.35 \mu \mathrm{m}, 3.3 \mathrm{VDC}$ CX3001 prototype performance results will soon be added to our database.

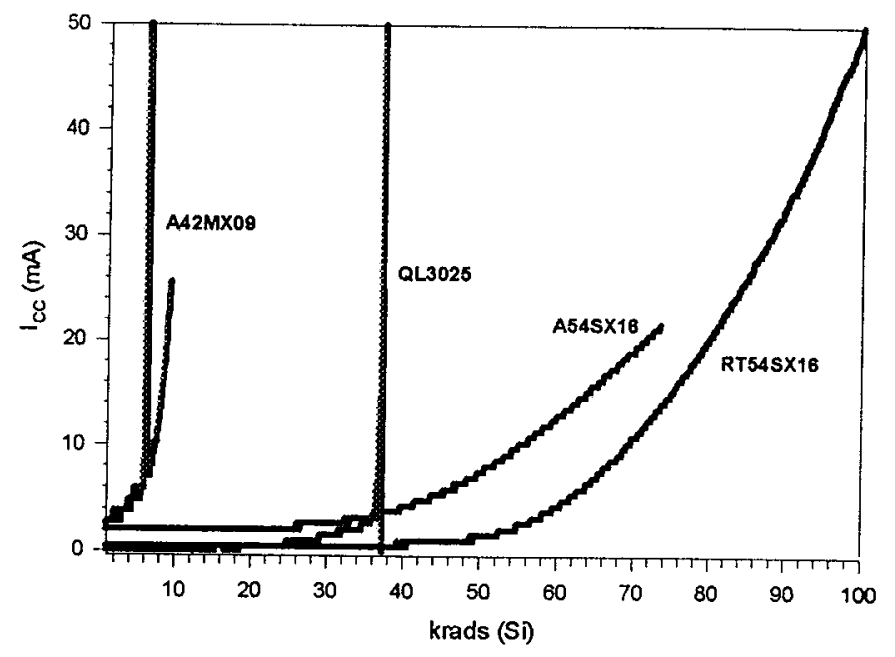

Figure 13. TD performance for new and prototype DUTs. COTS produced devices with a 3.3 volt core exhibited radiation-tolerant performance; a modified COTS device passed at $>100$ krads ( $\mathrm{Si}$ ).

Process modifications have been made to the prototype RT54SX16, with three splits of our test lot. Split 'A' was fabricated unmodified from the typical process, and lot splits ' $B$ ' and ' $D$ ' represent different levels of modification. A series of experiments validated the predictions, with improved total ionizing dose performance. With very conservative test standards and no annealing, lot split ' $\mathrm{B}$ ' passed at $80 \mathrm{krads}$ (Si) and lot split ' $D$ ' easily passed at the 100 krads (Si) level. This experiment shows the potential for a modified COTS part exhibiting radiation-hard, total dose performance.

Further testing has been conducted on the $0.8 \mu \mathrm{m}$ QYH500/Yamaha series. Previous testing [1] used laser programmed parts useful for rapid prototyping. Recent testing on two lots of "one-mask" devices, which are programmed with a single etch and are passivated, had performance levels of 15 to $30 \mathrm{krads}$ (Si). The same DUT logic design was put into the more architecturally attractive $0.6 \mu \mathrm{m} \mathrm{CX2041/Tower} \mathrm{device,} \mathrm{showing} \mathrm{a} \mathrm{total} \mathrm{dose}$ capability of approximately $7 \mathrm{krads}(\mathrm{Si})$. Based on the results of the prototype deep sub-micron FPGAs, we will next evaluate the $0.35 \mu \mathrm{m} \mathrm{CX3001/CSM} \mathrm{family.}$

\section{HARDENING EFFORTS}

\section{A. RH1020 and RH1280 Devices}

Over the last few years, $\mathrm{RH}$ series devices have been produced, using Lockheed-Martin Federal Systems as a foundry. Initially the commercial design was to be used, which kept the COTS design intact, achieving total dose hardness from the use of the LMFS process. While the A1020B $10 \mu \mathrm{m}$ epi device does latchup [12], qualification testing on the RH1020 $5 \mu \mathrm{m}$ epi process showed no sign of latchup and excellent total dose performance.

Based on the susceptibility of the ONO antifuse to rupture as a result of heavy ions [12], the antifuse thickness in the RH series was changed, decreasing the electric field strength which provides a more radiation-tolerant solution. Section III discussed the vulnerability of the RH1020's input buffer to single event transients and the improvements gained by redesigning the circuits. No effort was made to harden the storage elements with SEU performance ranging from low tolerance to "rad-tolerant" levels.

\section{B. CGaAs CLAy-10}

The goal of the AFRL/NASA/Motorola/SPEC complementary GaAs FPGA is to provide for low power, high speed, electronics for communication applications. Using $0.7 \mu \mathrm{m}$ low temperature technology, it is planned to achieve $350 \mathrm{MHz}$ performance using a $1.5 \mathrm{VDC}$ power supply with no SEL, an SEU LET $>20 \mathrm{MeV}-\mathrm{cm}^{2} / \mathrm{mg}$, and a total dose hardness exceeding 100 Mrads. The device is architecturally based on the re-programmable National Semiconductor CLAY-10 architecture.

\section{SOI AT6010}

This NASA/SNL/Honeywell/Atmel program will radiation-harden the AT6010 using a Honeywell silicon-oninsulator process. Goals for the program include a total dose hardness exceeding $200 \mathrm{krads}$ (Si), no SEL, and an SEU threshold of $>30 \mathrm{MeV}-\mathrm{cm}^{2} / \mathrm{mg}$ for both user storage and the configuration elements. This FPGA will be a $5 \mathrm{~V}$ device.

\section{D. $X Q R+000 X L$}

By special processing, the XQR4036XL, a $0.35 \mu \mathrm{m}$ device, as discussed in Section $\mathrm{V}$, has demonstrated a total dose capability of $60 \mathrm{krads}(\mathrm{Si})$ while meeting functional and parametric specifications. No latchup of the $7 \mu \mathrm{m}$ epi-based device was detected at an LET of $100 \mathrm{MeV}-\mathrm{cm}^{2} / \mathrm{mg}$ and a temperature of $125^{\circ} \mathrm{C}$ [13].

\section{E. RTSX and RHSX}

These $0.6 \mu \mathrm{m}$ devices are based on the commercial A54SX series and have had prototypes tested over the past two years. RTSX prototypes are produced at MEC and RHSX prototypes are fabricated at LMFS. As discussed in Section 
Pre-Print to be published in the IEEE Transactions on Nuclear Science, December, 1998

$\mathrm{V}$, total dose hardness for the RTSX can be made greater than 100 krads (Si) and the RHSX prototype easily passes $200 \mathrm{krads}(\mathrm{Si})$ [1]. A radiation-hardened antifuse structure has been demonstrated passing tests at a LET of $82.3 \mathrm{MeV}-\mathrm{cm}^{2} / \mathrm{mg}$. Present design modifications include the addition of the optional TRST! line to harden the IEEE JTAG 1149.1 TAP controller and a modified hard-wired flip-flop to increase SEU hardness from radiation-tolerant levels to radiation-hard. This device operates with a $3.3 \mathrm{VDC}$ core and is $5 \mathrm{~V}$-input tolerant.

\section{CONCLUSION}

Programmable devices will continue to be of increasing importance to spacecraft electronics designers as system requirements increase the trend towards higher performance electronics with increased processing bandwidth and shorter development times. These devices will be required for onboard processing of the increasing data volumes from sensors that cannot be accommodated by typical data storage and compression schemes with the available down link telemetry rates. State-of-the-art, commercial programmable devices have recently progressed rapidly down the technology curve, with operating frequencies rivaling that of high-powered discrete designs and ASICs, in many cases.

Programmable devices can be either custom-designed for space applications or rely on commercial technology and its associated infrastructure. While most programmable devices for space flight are derivatives of commercial designs, the LMFS PROM was designed with radiation issues in mind utilizing a low $(0.1 \mathrm{~V})$ bias across unprogrammed ONO antifuses to ensure high-reliability.

Commercially-derived devices' radiation tolerance range from poor to radiation-tolerant, in most cases. Some of the radiation hazards stem from structures and technologies that are perfectly reliable in the commercial/military sector, such as antifuses, TTL-compatible input buffers, flip-flop designs, and circuits such as the JTAG TAP controller, as examples. Total dose performance of $5.0 \mathrm{VDC}$ devices is highly variable, subject to process variations at the commercial foundries.

We have shown that devices' radiation performance levels can be increased to radiation-hard levels without the use of an expensive radiation-hard process. This is through a combination of modification of circuit designs and commercial processes. Examples include a radiationhardened antifuse and total dose performance greater than 100 krads (Si). A number of manufacturers are now actively modifying their designs and their foundries' processes to increase the radiation performance of commercially produced devices

Within the next few years, the commercially produced, modified devices will likely provide solid radiation performance for the majority of applications, including highspeed processing. Similarly designed devices, produced on traditional radiation-hardened lines, will be available for high levels of radiation performance.

\section{REFERENCES}

[1] R. Katz, K. LaBel, J.J. Wang, B. Cronquist, R. Koga, S. Penzin, and G. Swift, "Radiation Effects on Current Field Programmable Technologies, IEEE Transactions on Nuclear Science, NS-44. pp. 1945-1956 (1997).

[2] J.M. Benedetto, C. C. Hafer, A. R. Oliver, and D. B. Kerwin, "Amorphous Silicon Antifuse Programmable Array Logic Devices for High Reliability Space Applications," Government Microcircuits Applications Conference Digest of Papers, pp. 303-306, March 16-19, 1998, Arlington, VA.

[3] J. Banker, Pico Systems, Inc., private communication.

[4] C. Shih, R. Lambertson, F. Hawley, F. Issaq, J. McCollum, E. Hamdy, H. Sakurai, H. Yuasa, H. Honda, T. Yamaoka, T. Wada, and C. Hu, "Characterization and Modeling of a Highly Reliable Metal-to-Metal Antifuse for High-Performance and High-Density Field-Programmable Gate Arrays," $35^{\text {th }}$ Annual International Reliability Physics Proceedings, pp. 25-32 (1997).

[5] R. Wong, and E. Gordon, "Reliability Mechanism of the Unprogrammed Amorphous Silicon Antifuse," $32^{\text {nd }}$ Annual IEEE International Reliability Physics Proceedings, pp. 378382 (1994).

[6] J. Schwank, "Radiation Effects in Commercial Electronics," in 1994 IEEE NSREC Short Course, II-90-II-92.

[7] D. Mavis, and P. Eaton, "Temporally Redundant Latch for Preventing Single Event Disruptions in Sequential Integrated Circuits," a white paper by Mission Research Corporation (1998).

[8] R. Koga, S. Penzin, K. Crawford, and W. Crain, "Single Event Functional Interrupt (SEFI) Sensitivity in Microcircuits," Proceedings of the Fourth European Conference on Radiation and its Effects on Components and Systems, pp. 311-318 (1997).

[9] IEEE STD 1149.1-1990, IEEE Standard Test Access Port And Boundary-Scan Architecture, IEEE Computer Society (1993).

[10] K. LaBel, P. Marshall, J. Barth, R. Katz, R. Reed, H. Leidecker, and H. Kim, "Anatomy of an In-flight Anomaly: Investigation of Proton-Induced SEE Test Results for Stacked IBM DRAMs," accepted for publication in the IEEE Transactions on Nuclear Science, NS-45 (December 1998).

[11] R. Padovani, Xilinx Corporation, private communication.

[12] R. Katz, R. Barto, P. McKerracher, B. Carkhuff, and R. Koga, "SEU Hardening of FPGAs for Space Applications and Device Characterization," IEEE Transactions on Nuclear Science, NS-41, pp. 2179-2186 (1994.)

[13] P. Alfke, and R. Padovani, "Radiation Tolerance of HighDensity FPGAs," accepted for publication in the 1998 Military and Aerospace Programmable Logic Device Conference Proceedings, September 15-16, 1998, Greenbelt, MD. 
Pre-Print to be published in the IEEE Transactions on Nuclear Science, December, 1998

\section{APPENDIX I. SUMMARY OF DEVICES AND TECHNOLOGIES}

\begin{tabular}{|c|c|c|c|c|c|}
\hline Device & Series & Foundry & $\begin{array}{l}\text { Conflg. } \\
\text { Techn. }\end{array}$ & $\begin{array}{l}\text { Core } \\
\text { Volt. } \\
\end{array}$ & $\begin{array}{c}\text { Size } \\
(\mu \mathrm{m})\end{array}$ \\
\hline & $\mathrm{VF}-1$ & & SRAM & 2.5 & 0.18 \\
\hline $\mathrm{A} 40 \mathrm{MX02}$ & $40 \mathrm{MX}$ & $\mathrm{CSM}$ & ONO & 5 & 0.45 \\
\hline $\mathrm{A} 42 \mathrm{MX09^{1 }}$ & $42 \mathrm{MX}$ & $\mathrm{CSM}$ & ONO & 5 & 0.45 \\
\hline $\mathrm{A} 1020^{1}$ & Act 1 & MEC & ONO & 5 & 2.0 \\
\hline $\mathrm{A} 1020 \mathrm{~A}^{1}$ & Act 1 & MEC & ONO & 5 & 1.2 \\
\hline $\mathrm{A}^{1020 \mathrm{~B}^{1}}$ & Act 1 & MEC & ONO & 5 & 1.0 \\
\hline $\mathrm{A} 1280 \mathrm{~A}^{1}$ & Act 2 & MEC & ONO & 5 & 1.0 \\
\hline $\mathrm{Al} 4100 \mathrm{~A}^{\prime}$ & Act 3 & MEC & ONO & 5 & 0.8 \\
\hline $\mathrm{Al} 460 \mathrm{~A}^{1}$ & Act 3 & MEC & ONO & 5 & 0.8 \\
\hline A54SX16 ${ }^{1}$ & $s \mathrm{~S}$ & CSM & $\mathrm{M} 2 \mathrm{M}$ & 3.3 & 0.35 \\
\hline $\mathrm{AT} 28 \mathrm{C} 010^{2}$ & AT28C & Atmel & EEPROM & 5 & \\
\hline $\mathrm{AT}^{2} 610^{2}$ & AT6K & Atmel & SRAM & 5 & 0.8 \\
\hline
\end{tabular}

\begin{tabular}{|c|c|c|c|c|c|}
\hline Device & Series & Foundry & $\begin{array}{l}\text { Conffg. } \\
\text { Techn. }\end{array}$ & $\begin{array}{l}\text { Core } \\
\text { Volt. } \\
\end{array}$ & $\begin{array}{l}\text { Size } \\
(\mu \mathrm{m})\end{array}$ \\
\hline $\mathrm{C} \times 2041^{3}$ & $\mathrm{CX} 2001$ & Tower & METAL & $3.3,5$ & 0.6 \\
\hline $\mathrm{C} \times 3061^{3}$ & $\mathrm{CX} 3001$ & $\mathrm{CSM}$ & METAL & 3.3 & 0.35 \\
\hline JT22VP10 & PAL & Cypress & FUSE & 5 & \\
\hline QL24x32 & pASIC 1 & Cypress & $\mathrm{M} 2 \mathrm{M}$ & 5 & 0.65 \\
\hline QL3025 & pASIC3 & TSMC & M2M & 3.3 & 0.35 \\
\hline QYH $530^{3}$ & QYH500 & Yamaha & METAL & $3.3,5$ & 0.8 \\
\hline RH1020 & Act 1 & LMFS & ONO & 5 & 1.0 \\
\hline RH1 $1280^{1}$ & Act 2 & MEC & ONO & 5 & 0.8 \\
\hline RH $54 S \times 16^{1}$ & SX & LMFS & $\mathrm{M} 2 \mathrm{M}$ & 3.3 & 0.6 \\
\hline RT54SX16 ${ }^{1}$ & sX & MEC & $\mathrm{M} 2 \mathrm{M}$ & 3.3 & 0.6 \\
\hline UT22VP10 6 & RadPAL & UTMC & M2M & 5 & \\
\hline $\mathrm{XQR} 4036 \mathrm{XL}^{7}$ & $\mathrm{XC4000X \textrm {L }}$ & & SRAM & 3.3 & 0.35 \\
\hline
\end{tabular}

\section{Foundry Information}

CSM: Chartered Semiconductor Manufacturers

MEC: $\quad$ Matsushita Electric Company

TSMC: $\quad$ Taiwan Semiconductor Manufacturing Corp.

LMFS: Lockheed Martin Federal Systems

UTMC: United Technologies Microelectronics Center
Device Manufacturers

${ }^{1}$ Actel

${ }^{2}$ Atmel

${ }^{3}$ Chip Express

${ }^{4}$ Cypress

${ }^{5}$ Quicklogic

${ }^{6}$ UTMC

${ }^{7}$ Xilinx 
